\title{
Lesiones y alcohol: de la evidencia epidemiológica a la acción preventiva
}

\author{
Plasència, A. \\ Institut Municipal de Salut Pública de Barcelona y Departament de Pediatria, d'Obstetrícia i Ginecologia i de Medicina Preventiva, \\ i Salut Pública, Universitat Autònoma de Barcelona
}

Enviar correspondencia: Antoni Plasència. Institut Municipal de Salut Pública. Lesseps 1. 08023 Barcelona.

Tel.: 9323845 50. Fax: 9321731 97. E-mail: aplasenc@imsb.bcn.es

\section{RESUMEN}

Las lesiones suponen una de las principales causas de mortalidad, morbilidad y discapacidad a nivel mundial, y se prevé que se sitúen entre las causas que experimentarán un mayor incremento en los próximos 20 años. La implicación del alcohol en el riesgo de padecer una lesión ha sido demostrada en numerosos estudios, especialmente para el caso de las lesiones de tráfico, aunque también se ve con frecuencia implicado en las lesiones por caída, por fuego y por ahogamiento. En la Unión Europea se estima que anualmente se producen unas 10.000 muertes por lesiones de tráfico relacionadas con el alcohol ( 1 de cada 4 muertes de tráfico). El consumo de alcohol también se asocia a un mayor riesgo de ingreso hospitalario y a un mayor consumo de recursos sanitarios. En España -que se sitúa en el nivel intermedio de las tasas de mortalidad por lesión de tráfico atribuible al alcohol en Europa-, las estimaciones de presencia de alcohol en las defunciones de tráfico varían ampliamente, entre el 37 y el 77\%. La presencia de alcohol en los lesionados de tráfico atendidos en los servicios de urgencias hospitalarios muestra unos valores superiores a los límites legales en el 30 al $51 \%$ de los casos.

Existe abundante evidencia sobre la efectividad de las estrategias y programas de prevención de las lesiones relacionadas con el alcohol. Entre las más destacadas, se encuentran: el aumento de la edad mínima autorizada para el consumo de alcohol, las leyes de tolerancia cero y las que fijan los límites de alcohol en sangre por debajo de 0,8 gr/l, la reducción de los límites legales para los infractores, los programas preventivos a nivel comunitario, el cribado del alcohol y la intervención breve en los lesionados atendidos en urgencias, el consejo médico desde la consulta de atención primaria, la retirada del carnet de conducir y la inmovilización del vehículo.

Aunque no faltan las soluciones técnicas para hacer frente a este grave problema de salud pública, es necesario un debate que plantee la naturaleza esencialmente política y social de las decisiones, que deben implantarse de manera rápida y enérgica.

Palabras clave: lesiones, accidentes tráfico, urgencias, prevención.

\section{SUMMARY}

Injuries are one of the main causes of mortality, morbidity and disability in the world, and it is expected that they will undergo some of the largest negative increases in the next two decades. The implication of alcohol in the risk of an injury has been shown in many studies, especially for the case of traffic injuries, although this is also the case for injuries due to falls, fires and drowning. In the European Union alone, about 10,000 alcohol related injury deaths are estimated to occur yearly (1 in every 4 traffic injury deaths). Alcohol is also associated with a greater risk of hospital admission, and larger use of health care resources. In Spain -which shows intermediate alcohol related traffic mortality rates, estimates of blood alcohol levels (BAL) in fatal traffic injury cases vary substantially, between 37 and $77 \%$. BAL in non fatal traffic injury cases are beyond legal limits in 30 to $51 \%$ of the patients attended at emergency rooms.

There is a large amount of evidence regarding the effectiveness of strategies and programs to prevent alcohol-related injuries. Main ones include the raise of the legal minimal drinking age, zero tolerance laws, as well as laws setting maximum BAL below $0.8 \mathrm{gr} / \mathrm{l}$, the reduction of legal BAL for traffic offenders, community prevention programs, alcohol screening and brief interventions in the emergency rooms, medical counselling in primary care, suspension of driving license and vehicle immobilisation.

Despite the availability of technical solutions to face this serious public health problem, a debate is needed to put forward the essentially political and social nature of the decisions, which need to be rapidly and energically implemented.

Key words: Injuries, traffic accidents, emergencies, prevention. 


\section{LAS LESIONES COMO PROBLEMA DE SALUD GLOBAL}

$\mathbf{L}$ as lesiones -los mal llamados accidentes* - suponen una de las principales causas de mortalidad, morbilidad y discapacidad. En 1998, cerca de 5,8 millones de personas murieron en el mundo como resultado de alguna lesión', principalmente a causa del tráfico, de las caídas y de la violencia. Las lesiones causan el $15 \%$ de la carga global de enfermedad (global burden of disease), medida a través de los años de vida libres de discapacidad (DALYs) ${ }^{2}$. Su especial impacto en las edades jóvenes las sitúa entre las 10 principales causas específicas de DALYS. Además, el hecho de que los lesionados jóvenes sobrevivan cada vez más a lesiones que anteriormente causaban la muerte no hace sino incrementar el peso de las lesiones como causa de discapacidad permanen$t^{3}$. Las predicciones con respecto a las tendencias de dicho impacto no son menos negativas, estimándose que en el año 2020 las muertes por lesión afectarán a 8,4 millones de personas; las lesiones de tráfico pasarán a ser la segunda causa de mortalidad prematura, la tercera causa de discapacidad y la sexta causa de muerte general, siendo, con la infección por el VIH, la causa que experimentará un mayor incremento relativo ${ }^{4}$. Además, las lesiones de tráfico, por violencia y por las guerras se situarán entre las 15 causas más frecuentes de muerte prematura y de discapacidad, contrastando con causas como el sarampión, la malaria, las enfermedades diarreicas o las afecciones perinatales, que presentarán importantes reducciones, fruto de las acciones de prevención y control. Ello es una clara indicación de que las lesiones siguen siendo un problema de salud pública de primera magnitud, pero que no goza del necesario reconocimiento como una prioridad de las políticas de salud.

En Europa, el reflejo de esta situación se manifiesta en los cerca de 130.000 muertos que anualmente se producen por causas externas, destacando las lesiones en el hogar y en el tiempo de ocio (80.000 muertes) y de tráfico (40.000), junto con las 8.000 defunciones que se producen en el ámbito laboral. Más de 50 millones de europeos requieren anualmente atención sanitaria a causa de una lesión, de los cuales 40 millones son por lesiones en el hogar y en el tiempo de ocio, 3,5 millones se deben al tráfico rodado, y 10 millones resultan de eventos producidos en el trabajo. Aunque en la última década las muertes por lesión han ido descendiendo, parece que hay que atribuir esta tendencia más a los avances en la asistencia a los heridos que no a la reducción de su gravedad. Además, se observa un incremento de las víctimas de lesiones producidas en el hogar y en el tiempo de ocio, mientras que las tendencias de las lesiones de tráfico son muy dispares entre los distintos países de la Unión Europea.

En el caso de las lesiones en el hogar, los niños, los ancianos y los discapacitados son los grupos de población más vulnerables. Pero es en los mayores de 75 años en los que la tasa de mortalidad por lesiones es la más alta, por encima de los restantes grupos de edad, una circunstancia que, a la vista del creciente envejecimiento de la población europea, no hará sino aumentar. Con respecto a las lesiones infantiles, un reciente informe de UNICEF señala que las lesiones son la principal causa de muerte de los niños de 26 países de la OCDE -principalmente a causa del tráfico (41\%), seguido de los ahogamientos, los incendios, las caídas y los envenenamientos. Sin embargo, es de destacar que su

\footnotetext{
* El término lesión nos parece más adecuado que el de accidente, tanto por el hecho de que el primero se refiere al problema de salud como suceso relevante, como porque el segundo no permite diferenciar entre las circunstancias en las que se produce un daño corporal y su resultado. Por otra parte, el uso del término accidente refuerza la percepción, desgraciadamente aún muy extendida, de que las lesiones resultan de acontecimientos impredecibles, y por tanto no prevenibles, un aspecto que contradice un volumen cada día mayor de evidencia científica.
} 
impacto se ha reducido a la mitad entre 1970 y $1995^{5}$.

Se ha estimado que las lesiones en el hogar o durante las actividades de ocio suponen un coste de 230 billones de Euros al año en la UE. Por su parte, las lesiones de tráfico cuestan anualmente del orden de 165 billones de Euros, a los que hay que sumar los 20 millones de Euros que suponen las lesiones laborales, lo que implica un coste total anual que casi alcanza los 400 billones de Euros ${ }^{6}$.

\section{EL IMPACTO DE LAS LESIONES EN ESPA- $\tilde{\mathbf{N A}}$}

En el contexto europeo, España se sitúa entre los países en los que las lesiones de tráfico y del hogar tienen una mayor contribución al conjunto de lesiones, mientras que las lesiones por violencia (incluyendo agresiones, suicidios y autolesiones), tienen un peso relativo menor. Después del cáncer, las lesiones son la segunda causa más frecuente de mortalidad prematura medida en años potenciales de vida perdidos, tanto en hombres como en mujeres. Según la última Encuesta Nacional de Salud, en 1997 cerca del 9\% de la población española mayor de 15 años declaró haber sufrido algún accidente en el año anterior, porcentaje que se eleva a algo más del $10 \%$ en la población menor de 16 años. Casi un tercio de los accidentes se produjo en el hogar, seguido de la calle, en la que se reparten a partes iguales los accidentes de tráfico $(22 \%)$ y los de no de tráfico (22\%). El 82,6\% de los accidentes en adultos y el $86,1 \%$ de los producidos en los niños requirieron algún tipo de atención sanitaria y el $6,4 \%$ de los adultos y el $7 \%$ de los niños tuvieron que ser hospitalizados. Complementariamente, al menos el $44 \%$ de los adultos y el $49 \%$ de los niños sufrió una lesión de gravedad apreciable (fracturas, heridas profundas, envenenamientos y quemaduras). En conjunto, ello equivale a un número anual de más de 3.530 .000 víctimas, 2.950 .000 casos atendi- dos sanitariamente y 231.000 ingresos hospitalarios.

Las fracturas, luxaciones y traumatismos en general suponen una restricción de la actividad superior a 10 días en el 3,2\% de la población adulta española, situándose en un destacado segundo lugar entre las causas de restricción de la actividad, únicamente superados por el grupo de alteraciones asociadas al dolor, a la sobrecarga y al envejecimiento (artrosis, reumatismo, dolor de espalda, etc.). Ello implica una estimación mínima de más de 10 millones de días de actividad restringida anuales resultantes de alguna lesión.

Las lesiones de tráfico son la principal causa de muerte por lesiones y, en los hombres, se sitúan entre las 10 principales causas de muerte. Aunque su peso relativo se ha ido reduciendo en años recientes, causan cerca de una tercera parte de las muertes por traumatismo. Tras experimentar un fuerte crecimiento en los años 80 , en los 90 se inició un importante cambio de tendencia, con un descenso sostenido de las tasas. Sin embargo, esta tendencia se ha truncado nuevamente a partir de 1995, cuando se inicia un nuevo e ininterrumpido incremento de la mortalidad. Ello sitúa a España, junto con Portugal, Grecia y Francia, entre los países de la Unión Europea con un mayor impacto negativo de las lesiones de tráfico.

Con relación a las lesiones en el trabajo,

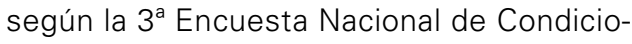
nes de Trabajo, un 8,8\% de la población asalariada española (el 10,6 \% de los hombres y el $5,4 \%$ de las mujeres) habría sufrido un accidente de trabajo durante el bienio 199697. Por sectores de actividad, las lesiones fueron más frecuentes en los servicios, seguidos de la industria, la construcción y la agricultura, siendo una parte apreciable de los mismos lesiones de tráfico.

En conjunto, las tendencias observadas indican que los Objetivos de Salud Para Todos en el 2000 fijados para España están lejos de haberse alcanzado, y muy especialmente para las lesiones de tráfico? 


\section{EPIDEMIOLOGÍA DE LAS LESIONES RELA- CIONADAS CON EL ALCOHOL}

La implicación del alcohol en el riesgo de lesiones ha sido demostrada en numerosos estudios $^{8}$. Sólo en los Estados Unidos de América, cerca de 50.000 muertes ocurren anualmente a causa de lesiones, tanto intencionales como no intencionales ${ }^{9}$. En la UE, se estima que se producen unas 10.000 muertes por lesiones de tráfico relacionadas con el alcohol (1 de cada 4 muertes de tráfico en Europa). El alcohol está relacionado con casi el $30 \%$ de los ingresos hospitalarios, especialmente en los servicios de urgencias ${ }^{10}$, así como con un mayor consumo de recursos sanitarios ${ }^{11}$.

Un reciente estudio de 14 países europeos $^{12}$ ha investigado si los cambios en el consumo de alcohol per cápita en los últimos 50 años se han asociado a cambios en la mortalidad por lesiones, analizando separadamente los países del sur de Europa (países "de vino"), los países del norte (países "de licores"), y los países de Europa central junto con la Gran Bretaña (países "de cerveza"). Los resultados indican que un incremento del consumo de alcohol en 1 litro se acompaña de un incremento medio de 2,65 muertes por 100.000 hombres debidas a una lesión y de 0,61 muertes por cada 100.000 mujeres. Sin embargo, dichos efectos son apreciablemente distintos según los países, siendo más notables en los países del norte, seguidos de los de Europa central y del sur. Para el caso de España, la asociación significativa fue de las más bajas de las observadas para los hombres $(1,19)$, siendo significativa únicamente en los grupos de mediana edad, mientras que no se encontró una relación en las mujeres. Los resultados sugieren que en los países del norte de Europa el $45 \%$ de las muertes por lesiones en hombres de 15 a 69 años se relacionan con el alcohol, mientras que esta cifra para los países del sur se estima en $40 \%$. Por otra parte, no puede olvidarse que el impacto negativo del alcohol se extiende a los niños, que también son vícti- mas de lesiones de tráfico con participación del alcohol ${ }^{13}$.

En España, las estimaciones equivalentes para el periodo 1981-1990 señalaron que las lesiones no intencionales suponían entre el 20 y el $25 \%$ de la mortalidad atribuible al alcohol $^{14}$, cifras similares a la estimada recientemente para Catalunya, que en 1997 fue del $23 \%{ }^{15}$.

El papel del alcohol ha sido más frecuentemente estudiado en las lesiones de tráfico, aunque también existe evidencia de su implicación en las caídas ${ }^{16}$, en las lesiones por fuego ${ }^{17}$ y por ahogamiento ${ }^{18}$. El problema de las lesiones es especialmente alarmante en los países de renta baja, en los que se observa un incremento del consumo de alcohol, y en los que se produce una incidencia especialmente elevada de lesiones, en ausencia de unas políticas activas de prevención y control. Por todo ello, el conocimiento de las características epidemiológicas de las lesiones en las que interviene el consumo de alcohol es un instrumento indispensable si se pretende atajar el oscuro panorama del impacto de las lesiones en la salud, tanto a nivel internacional como nacional, regional y local.

\section{Alcohol y lesiones de tráfico}

Existe una amplia evidencia científica de que la concentración de alcohol en sangre (CAS), incluso en niveles bajos, altera las habilidades de conducción e incrementa el riesgo de colisión, tal y como destaca una reciente revisión de 112 estudios $^{19}$. A niveles de 0,5 gr/l la mayoría de estudios encuentran una alteración significativa, valoración que se extiende al 94\% de los estudios para los niveles de 0,8 $\mathrm{gr} / \mathrm{l}$. Sin embargo, algunas habilidades empiezan a afectarse a niveles más bajos que otras; así, a CAS de 0,2 gr/l o menos, la capacidad de dividir la atención entre dos o más fuentes de información visual puede afectarse, mientras que con 0,5gr/l aparecen otras afectaciones, incluyendo el movimiento de los ojos, la resistencia al deslumbramiento, la percepción visual y el tiempo de reacción. 
El riesgo relativo de una colisión mortal en conductores con CAS positivas se incrementa con el aumento de dichas concentraciones, y de manera más marcada en los conductores jóvenes ${ }^{20}$. En CAS de entre 0,8 y 1 , el riesgo de colisión mortal varía entre el 11 por ciento (en conductores mayores de 35 años) y el 52 por ciento (en conductores masculinos de entre 16 y 20 años). Estos efectos también han sido puestos de manifiesto en otros tipos de conductores, como pilotos de avión o de barco. Por otra parte, el consumo de alcohol también se relaciona con un riesgo de hasta 2,5 veces de volver a sufrir una lesión de tráfico, ya sea con resultado de muerte o de reingreso hospitalario ${ }^{21}$, así como con una mayor gravedad de las lesiones $^{22}$ y un mayor riesgo de complicaciones ${ }^{23}$.

El alcohol puede actuar a nivel celular dificultando la recuperación post-lesional, afectando a la supervivencia. Puede también interferir en el sistema nervioso, afectando a la respuesta ante el shock y al reflejo tusígeno, lo que en determinadas circunstancias puede conllevar un mayor riesgo de muerte.

Diversos factores han demostrado modificar la influencia del alcohol en la conducción, entre los que destaca la tolerancia al alcohol, la edad, el género y la falta de sueño. Así, parece que la realización repetitiva de determinadas tareas de conducción bajo la influencia del alcohol pueda hacer que algunas personas sean menos sensibles a la afectación de sus habilidades, aunque dicha circunstancia deja de ser cierta cuando estas personas realizan tareas nuevas o que plantean demandas inesperadas. Por otra parte, a iguales CAS, las edades más extremas (los conductores menores de 20 años y los mayores de 85 años) se asocian a un mayor riesgo de colisión mortal, de entre 4 y 8 veces, respectivamente, que los conductores de 20 a 69 años $^{24} \mathrm{En}$ los conductores masculinos de menos de 21 años un incremento de CAS de 0,2 aumenta en más del doble el riesgo de colisión por salida de la vía. Por su parte, a igual CAS, las mujeres tienen un menor riesgo de colisión que los hombres. El mayor riesgo que se observa en los jóvenes se atribuye a la falta de experiencia así como al exceso de autoconfianza, una circunstancia que se agudiza con la presencia como pasajeros de otros jóvenes con CAS positivas ${ }^{25}$. En las personas mayores, el mayor riesgo de lesión de tráfico se asocia con alteraciones sensoriales y de la atención, observándose que los conductores mayores con antecedentes de alcoholismo tienen un mayor riesgo de colisión. Con respecto al sueño, se sabe que CAS de 0,1 ya incrementan la susceptibilidad a la somnolencia y el consiguiente riesgo de colisión.

Un reciente estudio del impacto del alcohol en la mortalidad por lesiones de tráfico en distintos países europeos indica para el conjunto de los países una asociación positiva entre las tendencias del consumo de alcohol per cápita y la mortalidad de tráfico, aunque en el caso de España no se encontró dicha asociación ${ }^{26}$. En Estados Unidos se ha estimado que 3 de cada 10 ciudadanos resultarán a lo largo de su vida implicados en alguna colisión relacionada con el alcohol ${ }^{27}$.

En el caso de España, las estimaciones de presencia de alcohol en las defunciones por lesiones de tráfico varían ampliamente, entre el 37 y el $77 \%$, con niveles superiores a los límites legales en el 35 al $40 \%$ de los mismos, aunque es complejo determinar si estas variaciones se deben a cambios en la implicación del alcohol o a diferencias metodológicas en los distintos estudios ${ }^{28}$. La última Memoria del Instituto de Toxicología ${ }^{29}$ relativa a 1613 muertes en accidentes de tráfico en el año 2000 (1363 conductores y 250 atropellados, que suponen el $32 \%$ de las muertes comunicadas por la Dirección General de Tráfico para ese año) señala que el $41 \%$ de los conductores fallecidos analizados presentaron alcoholemias positivas (79\% por encima de los 0,5 g/l), siendo superior en hombres (43\%) y en conductores de coche (36\%). La prevalencia de CAS positiva no es sustancialmente distinta de la de años anteriores (43\% en 1998 y $37 \%$ en 1999). Destaca además que la presencia de alcohol se acompañó de indicios de alguna droga en el $10 \%$ de los casos positivos, siendo la cocaína la más frecuente, en el $67 \%$ de 
los mismos. Por otra parte, el $39 \%$ de los peatones atropellados analizados presentó indicios de CAS, siendo superior en hombres (44\%); cerca del $80 \%$ de los peatones positivos presentó una CAS superior a los 0,5 g/l. La prevalencia de CAS en peatones fallecidos es más elevada que en anteriores años (32\% en 1998 y 34\% en 1999). Una limitación relevante es la ausencia de especificación de la información relativa a los ocupantes de motos y ciclomotores, que en España suponen una parte significativa de las víctimas del tráfico ${ }^{30}$. Tampoco existe información sobre el papel del alcohol en las lesiones resultantes de actividades náuticas de recreo, a pesar de la importancia de las mismas en nuestro país, y de las evidencias al respecto publicadas en otros países ${ }^{31}$.

Cabe señalar que en el contexto europeo, España se sitúa en el nivel intermedio de las tasas de mortalidad por lesión de tráfico atribuible al alcohol (24 por 100.000), contrastando con las cifras de Alemania $(50,7)$, Suiza $(40,2)$, Austria $(30,9)$ y Dinamarca $(29,7)^{32}$, aunque no puede descartarse que las diferencias se deban a sesgos de selección según los países en la medida de CAS en los fallecidos debidos al tráfico.

La presencia de CAS en los lesionados atendidos en los servicios de urgencias hospitalarios en España muestra unos valores superiores a los límites legales en el 30 al $51 \%$ de los $\operatorname{casos}^{28}$. Por otra parte, la realización de alcoholemias por las policías de tráfico arroja un porcentaje de positividad del $4 \%{ }^{33}$. El riesgo relativo entre la CAS y la presentación de una lesión de tráfico se ha estimado entre 7,5 y 55,8, según las áreas geográficas y categorías de consumo comparadas ${ }^{34,35}$. Estas cifras son consistentes con la observación de que el $60 \%$ de los conductores es bebedor habitual, siendo el 7\% AUDIT positivo, y habiéndose diagnosticado abuso o dependencia del alcohol en el $2 \%{ }^{36}$.

\section{Alcohol y lesiones por caídas o golpes}

Se ha señalado que a CAS de 0,1 gr/l se empieza a manifestar claros signos de des- equilibrio. Ello, junto con la alteración de la concentración y de los reflejos, provoca que la presencia de CAS también se asocie a caídas que pueden resultar en lesiones. Se ha estimado que el alcohol está presente en el 21 al $77 \%$ de las muertes por caídas y en el 18 al $53 \%$ de las lesiones por dicha causa ${ }^{37}$. Un estudio en Finlandia encontró que el riesgo relativo de caídas en individuos con CAS de 0,5 a $0,1 \mathrm{gr} / \mathrm{l}$ era 3 veces mayor que en los individuos sin alcohol, y hasta 60 veces mayor con CAS de 0,16 gr/l o más ${ }^{38}$. Asimismo, el estudio de las tendencias de la mortalidad por caídas en 14 países europeos muestra una relación variable con las tendencias del consumo de alcohol ${ }^{26}$; así, en el norte de Europa la asociación fue significativa en los hombres, pero no en las mujeres, relación que también se encontró -aunque de manera menos marcada- para los países de Europa central; sin embargo, en los países del sur de Europa -entre ellos, España, no se encontró ninguna relación entre la mortalidad por caídas y el consumo de alcohol.

\section{Alcohol y otras causas de lesión}

Además del tráfico y de las caídas, los ahogamientos y las lesiones debidas al fuego son las lesiones no intencionales que más se asocian al consumo de alcohol ${ }^{39}$. Así, entre el 21 y el $50 \%$ de las muertes por ahogamiento se han atribuido al alcohol38,40,41, cifras que se sitúan entre el 9 y el $86 \%$ para el caso de las muertes resultantes de incendios ${ }^{42}$. Los muertos por quemaduras tenían un riesgo mayor de haber consumido alcohol que las víctimas no mortales de quemaduras. En el caso de los ahogamientos, el consumo de alcohol se asocia a baños nocturnos, en solitario, y a actividades náuticas sin uso de dispositivos de flotación. Además, el alcohol puede crear una falsa sensación de calor que puede resultar en una prolongación excesiva del tiempo de nado, con el riesgo de hipertensión venosa, taquicardia y colapso cardiovascular. 


\section{POLÍTICAS Y PROGRAMAS DE PREVEN- CIÓN DE LAS LESIONES RELACIONADAS CON EL ALCOHOL}

Existe abundante literatura internacional sobre la efectividad de las estrategias de prevención de las lesiones relacionadas con el consumo de alcohol, especialmente para el caso de las lesiones de tráfico. Lamentablemente, esta evidencia es prácticamente ausente para el caso de España, donde no se han publicado evaluaciones del impacto en la incidencia de las lesiones de las acciones de prevención y control del consumo de alcohol. Por tanto, revisaremos aquí, de manera breve, las estrategias que han demostrado ser efectivas en otros países, algunos de los cuales llevan décadas de experiencia en este campo.

Aumento de la edad mínima autorizada para el consumo de alcohol

En los Estados Unidos, se ha estimado que el incremento de la edad a los 21 años ha reducido en un $13 \%$ las muertes de tráfico en los conductores de 18 a 20 años, con una disminución significativa de las colisiones, incluidas las mortales. Aunque el cumplimiento de las normas que prohiben la venta de alcohol a menores no siempre es elevado, los esfuerzos comunitarios para incrementar dicho cumplimiento pueden ser efectivos ${ }^{43}$.

\section{Leyes de tolerancia cero}

Estas leyes fijan como límites de CAS valores de 0,0 a 0,2 gr/l para los conductores jóvenes (generalmente, menores de 21 años). Su aplicación ha resultado en una disminución del $20 \%$ en la proporción de conductores jóvenes con CAS positivas implicados en colisiones mortales y en colisiones nocturnas por salida de la vía ${ }^{44}$. Asimismo, se ha documentado que los jóvenes conductores parecen aceptar mejor que sus mayores la separación de la conducción y del consumo de alcohol, una circunstancia que se ha atribuido a la aplicación de las leyes de tolerancia cero ${ }^{45}$.
Leyes que fijan los límites de CAS por debajo de $0,8 \mathrm{gr} / \mathrm{l}$

Existe evidencia de que una reducción de los niveles de CAS favorece una reducción del impacto del alcohol en el tráfico. Por ello, Suecia ha fijado los límites máximos más bajos de la Unión Europea $(<0,2 \mathrm{gr} / \mathrm{l})$, habiéndose producido recientes reducciones en los límites autorizados por diversos países, entre ellos España, fijados en 0,5gr/l para los conductores en general, y $0,3 \mathrm{gr} / \mathrm{l}$ para los principiantes.

Reducción de los límites legales de CAS para los infractores por conducción bajo los efectos del alcohol y para los transportistas

La reducción de los límites legales para los infractores parece haber disminuido la frecuencia de colisiones mortales en dicha población ${ }^{46}$. Dicha aplicación en los conductores profesionales -como en el caso de España- parece también tener un imapcto favorable en el riesgo de colisión.

\section{Programas preventivos a nivel comunitario}

Las acciones comunitarias orientadas a reducir el consumo de alcohol en la conducción, combinando esfuerzos desde el ámbito de la salud pública y de las organizaciones ciudadanas, a través de campañas mediáticas, formación policial, educación en las escuelas e institutos, y vigilancia de los puntos de venta de bebidas alcohólicas, se acompañan de una disminución de las colisiones mortales, incluyendo las relacionadas con la presencia de CAS, así como de una disminución de las lesiones de tráfico ${ }^{47,48}$. Existe un volumen creciente de recursos disponibles de apoyo a dichos programas ${ }^{49}$, algunos de los cuales han sido objeto de una revisión sistemática sobre su efectividad ${ }^{50}$, identificando cuatro intervenciones efectivas para la reducción de la conducción bajo los efectos del alcohol: leyes que limitan los niveles de alcohol en la población general, leyes que reducen dichos niveles para los conductores jóvenes o noveles, leyes que elevan la edad mínima de consumo a los 21 años y programas de control aleatorio de alcohole- 
mias en las vías de circulación (sobriety check-points). Debemos recalcar que las campañas informativas pueden incrementar la información y la concienciación, aunque no son por sí mismas efectivas si no se acompaña de un conjunto de acciones complementarias a nivel comunitario ${ }^{51}$. A éstas cabe añadir la implantación de programas de "conductores designados" (designated driver), mediante los cuales se designa a una persona de entre un grupo de unos pocos bebedores que acepta no consumir alcohol y conducir al resto del grupo de vuelta a sus domicilios en condiciones seguras ${ }^{52}$.

\section{Cribado del alcohol e intervención breve en} los lesionados atendidos en urgencias

Los pacientes atendidos en los servicios de urgencias como resultado de una lesión de tráfico pueden tener una mayor motivación para cambiar su conducta en relación con el consumo de alcohol ${ }^{53}$. La aplicación de intervenciones breves en el marco asistencial muestran una reducción del consumo subsiguiente y del riesgo de reingreso hospitalario, así como de la conducción bajo los efectos del alcohol, de las infracciones de tráfico y de los problemas relacionados con el consuno de alcohol en los más jóvenes ${ }^{54,55}$. En España, se ha iniciado de manera piloto este tipo de intervención en un centro hospitalario de Barcelona ${ }^{56}$.

Consejo médico desde la consulta de atención primaria

La evidencia disponible apunta a que el médico de cabecera puede ayudar desde su consulta a identificar los bebedores de riesgo y ofrecerle una intervención breve ${ }^{57}$, siendo una de las recomendaciones de la Preventive Services Task Force de Estados Unidos ${ }^{58}$. En España, cabe destacar las recientes acciones de promoción de esta estrategia, tanto por parte del Programa PAPPS de la SEMFYC ${ }^{59}$, como de algunas administraciones sanitarias ${ }^{60}$.

\section{Retirada del carnet de conducir}

Las leyes que obligan a la retirada del carnet en caso de infracción por consumo de alcohol durante la conducción se acompañan de una reducción en las colisiones mortales con presencia de alcohol, así como de las infracciones repetidas por conducir bajo los efectos del mismo. Sin embargo, se ha visto que un porcentaje importante de conductores con suspensión del carnet continúan conduciendo, lo que puede obligar a introducir medidas que ayuden a facilitar su control, como la introducción de adhesivos especiales en las matrículas de sus vehículos.

\section{Inmovilización del vehículo}

Las estrategias de inmovilización o de incautación del vehículo de aquellos conductores con infracciones repetidas por conducción bajo los efectos del alcohol han mostrado una menor recurrencia de las conductas infractoras por parte de dicha población ${ }^{61}$.

\section{Otras estrategias preventivas}

Aunque se han publicado algunos resultados prometedores, la utilización de bloqueadores del encendido del motor en caso de CAS elevadas -un dispositivo instalado en el vehículo que mide el alcohol expirado y que impide, en caso de superar un determinado nivel, el encendido del motor- muestra una evidencia controvertida sobre su impacto sostenido en la reducción de nuevas infracciones, especialmente una vez el dispositivo es retirado cuando ha transcurrido el periodo de sanción ${ }^{62}$. Asimismo, se encuentran en curso de evaluación algunos programas de educación dirigidos al personal expendedor de bebidas alcohólicas. Por su parte, las intervenciones de educación sanitaria en el medio escolar pueden tener una contribución positiva, en el marco de programas que aborden de forma amplia diversos factores de riesgo para la salud ${ }^{63}$. Finalmente, algunas intervenciones en el campo fiscal, como el incremento de los impuestos sobre el alcohol, con la consiguiente elevación de los precios, son probablemente las medidas que pueden tener un mayor impacto en los hábitos de consumo de los jóvenes ${ }^{64}$.

Algunas de las estrategias descritas para la prevención de las lesiones de tráfico relacionadas con el alcohol también pueden ser efectivas para prevenir las lesiones por caída, 
por ahogamiento y por quemaduras, tanto el marco de las acciones de prevención del consumo de alcohol en la población general, como a través de programas dirigidos específicamente a subgrupos de población y en entornos de especial riesgo ${ }^{65}$.

\section{CONCLUSIONES}

Se ha dicho que el alcohol es a las lesiones como el Virus de Inmunodeficiencia Humana al sida: disminuye las defensas y aumenta el riesgo de enfermedad ${ }^{66}$. La magnitud del impacto negativo de las lesiones en el mundo y las muy desfavorables perspectivas de su tendencia en las próximas décadas obligan a ser mucho más enérgicos en el reconocimiento de este grave problema de salud pública y en la implantación de las estrategias que han demostrado ser efectivas para su prevención y control.

En el caso de España, son abundantes los datos epidemiológicos que confirman la importancia del problema, especialmente con respecto al alcohol y las lesiones de tráfico. Aunque en los últimos años se han tomado medidas preventivas en la dirección deseable, como la reducción de los niveles legales máximos de CAS en la conducción, el resto de las estrategias de probada efectividad distan mucho de ser generalizadas y de aplicarse con rigor. Es especialmente importante que se plantee de manera clara la relevancia de introducir - iy hacer cumplir!- medidas más restrictivas con respecto al consumo y a los niveles legales de alcohol en los menores de 21 años, y que se desarrollen intervenciones administrativas y judiciales específicamente dirigidas a los infractores. Asimismo, el sistema sanitario, y en especial los servicios de urgencias, la atención primaria y los centros de reconocimiento de conductores, no pueden mantenerse ajenos al problema, como eslabones clave que pueden ser de algunas de las acciones de prevención, tanto primaria como secundaria. Finalmente, deben dinamizarse programas de acción comunitaria que utilicen estrategias múltiples de actuación, contando con la participación de entidades ciudadanas y del mundo de la empresa. En apoyo de este conjunto de actuaciones, también habrá que seguir profundizando en la exhaustividad y calidad de la información en las víctimas de lesiones, así como dotar de una financiación adecuada los proyectos de investigación y evaluación de todas aquellas estrategias que puedan suponer un avance en la prevención de las lesiones relacionadas con el alcohol. Las soluciones técnicas no faltan, pero es necesario un debate que plantee la naturaleza esencialmente política y social de las decisiones que deben tomarse para hacerlas efectivas. La ciencia está servida, pero sigue faltando el empeño en trasladar la evidencia epidemiológica a la acción preventiva.

\section{BIBLIOGRAFÍA}

(1) World Health Statistics, 1999. Geneva: World Health Organization, 2000.

(2) Murray CJL, Lopez AD. Global mortality, disability, and the contribution of risk factors: Global Burden of Disease Study. Lancet 1997; 349: 1436-42.

(3) Jarvis S, Pless B. The continuing global challenge of injury. BMJ 2001; 322:1557-58.

(4) Murray CJL, Lopez AD. Alternative projections of mortality and disability by cause 1990-2020: The Global Burden of Disease Study. Lancet 1997;349:1498-1504.

(5) Unicef. A league table of child deaths by injury in rich nations. Innocenti report card No.2. Florence: Unicef Innocenti Research Centre,2001.

(6) Accidents and injuries: the problem and the scope for improvement. European Public Health Update 2001;56:2-3.

(7) Plasència $A$, Moncada S. Objetivo 11: reducir los accidentes: en: Alvarez-Dardet C, Peiró S, eds. La salud pública ante los desafíos de un nuevo siglo. Informe SESPAS 2000. Granada: Sociedad española de Salud Pública y Administración Sanitaria; 165-74.

(8) Brismar B, Bergman B. The significance of alcohol for violence and accidents. Alcoh Clin Exp Res 1998; 22:229S-306S. 
(9) Alcohol and unintentional injury. Fact sheet. Alcohol Related Injury and Violence. (www.tf. org/tf/alcohol/ariv/facts/injufct5.html)

(10) Cherpitel C. Alcohol and injuries: a review of international emergency room studies. Addiction 1993;88:923-37.

(11) Blose JO, Holder HD. Injury-related medical care utilization in problem drinking population. Am J Public Health 1991;81:1571-75.

(12) Skog OJ. Alcohol consumption and overall accident mortality in 14 European countries. Addiction 2001; 96(S1):S35-S47.

(13) Li G. Child injuries and fatalities from alcoholrelated motor vehicle crashes. JAMA 2000; 283:2291.

(14) Prada Puentes C, del Rio Gracia MC, Yanez JL, Alvarez Gonzalez FJ. Mortalidad relacionada con el consumo de alcohol en España: 19811990. Gac Sanit 1996; 10:161-8.

(15) Farreny M, Godoy P, Revuelta E. Mortalidad atribuible al alcohol en Cataluña y sus provincias. Aten. Primaria 2001;27:318-23.

(16) Hingson R, Howland J. Alcohol as a risk factor for injury or death resulting from accidental falls: a review of the literature. J Stud Alcohol 1987; 48:212-19.

(17) Leth P, Gregersen M, Sabroe S. Fatal residential fire accidents in the municipality of Copenhagen 1991-1996. Prev Med 1998; 27:444-51.

(18) Lunetta P, Pentilla A, Sarna S. Water traffic accidents, drowning and alcohol in Finland 19691995. Int J Epidemiol 1998;27:1038-43.

(19) Moskowitz H, Fiorentino D. A review of the literature on the effects of low doses of alcohol on driving-related skills. Washington, DC: National Highway Traffic Safety Administration, 2000

(20) Zador PL, Krawchuck SA, Voas RB. Alcoholrelated relative risk of driver fatalities and driver involvement in fatal crashes in relation to driver age and gender. An update using 1996 data. J Stud Alcohol 2000;61:387-95.

(21) Rivara FP, Koepsell TD, Jurkovitch GJ, Gurney JG, Soderberg R. The effects of alcohol abuse on readmission for trauma. JAMA 1993;270:1962-64.

(22) Tulloh BR, Collopy BT. Positive correlation between blood alcohol level and ISS in road trauma. Injury 1994;25:539-43.

(23) Brewer RD, Morris PD, Cole TB, Watkins S, Patetta MJ, Popkin C. The risk of dying in alco- hol related automobile crashes among habitual drunk drivers. N Engl J Med 1994;331:513-17.

(24) Traffic Safety Facts 1999: Young drivers. Washington, DC: National Highway Traffic Safety Administration, 2000.

(25) Preusser DF, Ferguson SA, Williams AF. Effect of teenage passengers on the fatal crash risk of teenage drivers. Accid Anal Prev 1998;30:217-22.

(26) Skog AJ. Alcohol consumption and mortality rates from traffic accidents, accidental falls and other accidents in 14 European countries. Addiction 2001; 96(S1):S49-S58.

(27) Alcohol-related injuries. Fact Book for the Year 2000. National Center for Injury Prevention and Control, Centers for Disease Control (www. cdc.gov/ncipc/pub-res/factbook/alcohol.htm)

(28) Plasència A. Bases epidemiológicas para las políticas de prevención de los accidentes de tráfico relacionados con el alcohol en España. Adicciones 1999;11:229-36.

(29) Instituto de Toxicología. Memoria Análisis Toxicológico. Muertes en accidentes de tráfico. Año 2000. Madrid: Ministerio de Justicia, 2001.

(30) Plasència A, Borrell C, Antó JM. Emergency department and hospital admissions and deaths from traffic injuries in Barcelona, Spain. A one-year population-based study. Accid Anal Prev 1995;27:591-600.

(31) Smith GS, Kraus JF. Alcohol and residential, recreational, and occupational injuries: a review of the epidemiologic evidence. Annu Rev Public Health 1988;9:99-121.

(32) The European Perspective-Costs of alcohol. Alcohol Concern Factsheets. Factsheet 28. (www.alcoholconcern.org.uk/information/factsheets/factsheet20.htm)

(33) Consejo Superior de Tráfico y Seguridad de la Circulación Vial. Plan Nacional de Seguridad Vial para el año 2001. Madrid: Ministerio del Interior, 2001.

(34) Cía MT, Arévalo JM, Ardanaz E, Barricarte A, Chueca P, Serrano S. Nivel de alcohol y riesgo de lesión por accidente de tráfico en Tudela (Navarra). Gac Sanit 1996;53:55-61.

(35) Sesma FJ, Ardanaz E, Lera JM, Belzunegui T, Sola A, Gómez I. El riesgo de lesión en accidente de tráfico por conducción bajo los efectos del alcohol en Navarra. Gac Sanit 1992;6:117-22 
(36) Del Río MC, González-Luque JC, Alvarez FJ. Alcohol-related problems and fitness to drive. Alcohol 2001 36: 256-261.

(37) Hingson R, Howland J. Alcohol and non-traffic unintended injuries. Addiction 1993;88:877-83.

(38) Alcohol and health-implications for public health policy. Report of a WHO Working Group. Oslo, 9-13 October 1995 (en: www.who.dk/hp/ oslo.htm)

(39) Smith G, Branas CC, Miller TR. Fatal nontraffic injuries involving alcohol: a metanalysis. Ann Emerg Med 1999;33:659-68.

(40) Wintemute GJ, Teret SP, Kraus JF, Wright M. Alcohol and drowning: an analysis of contributing factors and a discussion of criteria for case selection. Accid Anal Prev 1990;22:291-96.

(41) Howland J, Hingson R. Alcohol as a risk factor for drownings: a review of the literature (19501985). Accid Anal Prev 1988;20:19-25.

(42) Barillo D, Goode R. Substance abuse in victims of fire. J Burn Care Rehab 1996;17:71-76.

(43) Voas RB, Tippetts AS. The relationship of alcohol safety laws to drinking drivers in fatal crashes. National Highway Safety Administration, 1999. (www. nhtsa.dot.goc/ people/ injury/ alcohol)

(44) Hingson R, Heeren T, Winter M. Lower legal blood alcohol limits for young drivers. Public Health Rep 1994;109:738-44.

(45) Roeper PJ, Voas RB. Underage drivers are separating drinking from driving. Am J Public Health 1999;89:755-57.

(46) Hingson R, Heeren T, Winter M. Effects of Maine's $0.05 \%$ legal blood alcohol levels for drivers with DWI convictions. Public Health Rep 1998;113:440-46.

(47) Hingson R, McGovern T, Howland J et al. Reducing alcohol impaired driving in Massachussetts: The Saving Lives Program. Am J Public Health 1996;86:791-97.

(48) Holder HD, Gruenewald PJ, Ponicki WR et al. Effect of community-based interventions on high-risk drinking and alcohol-related injuries. JAMA 2000;284:2341-47.

(49) Impaired Driving Division, National Highway Traffic Safety Agency (www.nhtsa.dot.gov/people/injury/alcohol).

(50) Task Force on Community Preventive Services. Motor-vehicle occupant injury: strategies for increasing use of child safety seats, increasing use of safety belts, and reducing alcohol-impaired driving. MMWR 2001;50:1-13.

(51) Holder H. Mass communication as an essential aspect of community prevention to reduce alcohol in traffic crashes. Alcohol Drugs Driving 1994;10:295-307.

(52) A Guide to developing a community-based designated driver program. National Highway Traffic Safety Administration (www.ncadd. com/designated)

(53) DiClemente CC, Bellino LE, Neavins TM. Motivation for change and alcoholism treatment. Alcohol Res Health 1999;23:86-92.

(54) Gentilello LM, Rivara FP, Donovan DM et al. Alcohol interventions in a trauma center as a means of reducing the risk of injury recurrence. Ann Surg 1999;230:473-83.

(55) Monti PM, Colby SM, Barnett NP et al. Brief intervention for harm reduction with alcohol positive older adolescents in a hospital emergency department. J Consult Clin Psychol 1999;67:989-94.

(56) Rodriguez-Martos, Plasència A, Escayola M, Martí J, Torralba LL. Intervención breve sobre accidentados con alcoholemia positiva desde un centro de traumatología. Adicciones $2001 ; 13($ S1):47-48.

(57) Fleming MF, Barry KL, Manwell LB, Johnson K, London R. Brief physician advice for problem alcohol drinkers. A randomized controlled trial in community based primary care practices. JAMA 1997;277:1039-45.

(58) Counseling to prevent motor vehicle-injuries. Guide to clinical preventive services. 2nd ed. Baltimore, MD: Williams and Wilkins, 1996; 643-58.

(59) Córdoba García R, Ortega Sánchez-Pinilla R, Cabezas Peña C, Forés García D, Nebot Adell M. Recomendaciones sobre el estilo de vida. Aten. Primaria 1999;24(S1):118-32.

(60) Consell preventiu sobre seguretat viària a l'atenció primària: recomanacions per a la seva extensió. Pla de Salut. Quadern núm.13. BarceIona: Departament de Sanitat i Seguretat Social, Generalitat de Catalunya, 2000.

(61) Voas RB, Tippetts A, Taylor E. Temporary vehicle impoundment in Ohio: a replication and confirmation. Accid Anal Prev 1998;30:651-56.

(62) Beck KH, Rauch WJ, Baker EA, Williams AF. Effects of ignition interlock license restrictions on drivers with multiple alcohol offences: a ran- 
domized trial in Maryland. Am J Public Health 1999;89:1696-1700.

(63) May C. Research on alcohol education for young people: a critical review of the literature. Health Educ J 1991;50:195-99.

(64) Ponicki W, Hoder HD, Gruenewald PJ, Romelsjo A. Altering alcohol price by ethanol content: results from a Swedish tax policy in 1992. Addiction 1997:92:859-70.

(65) Dinh-Zarr, DiGuiseppi C, Hetiman E, Roberts I. Interventions for preventing injuries in problem drinkers. Cochrane Database Syst Rev 2000; 2:CD001857.

(66) Foege WH. Highway violence and public policy. N Engl J Med 1987;316:1407-8. 